\section{Ciencia de Implementación en la evaluación de programas y servicios de salud en Chile}

\section{Implementation science to evaluate health programs and services}

\section{Señor Editor,}

El programa de Salud del actual gobierno incluye propuestas que consideran, entre otras, 1) la inclusión de nuevos modelos de gestión para mejorar eficiencia y calidad; 2) la incorporación de tecnologías que maximicen la atención clínica; 3) la reorganización de los servicios de salud, incluyendo infraestructura y recursos humanos; y 4) la implementación (o re implementación) de programas nacionales para prevenir obesidad o consumo de sustancias ${ }^{1}$. Estas propuestas parecen, al menos en el escrito, atingentes y necesarias para un país como Chile, que realiza escasa evaluación de procesos y resultados de su oferta programática en Salud. Poco es descrito respecto de las estrategias a utilizar para que dichos planteamientos se lleven a la práctica de buena manera. Además, se desconoce si tales propuestas consideran un enfoque de evidencia científica y transferencia de conocimiento, en concordancia con las recomendaciones de la Organización Mundial de la Salud (OMS) y la Organización para la Cooperación y el Desarrollo Económico (OCDE).

Chile tiene una extensa historia de reformas sanitarias exitosas. El desarrollo de servicios de salud inspirados en un modelo de atención en red y con un fuerte arraigo en atención primaria ha permitido asegurar cobertura y acceso a buena parte de la población. Entre las reformas de mayor relevancia se encuentra el régimen de garantías explícitas de salud (GES), cuyo propósito es proveer servicios basados en la evidencia para las personas afectadas por patologías prioritarias. Dicha estrategia representa uno de los primeros intentos sistemáticos de institucionalizar el uso de la mejor evidencia científica para abordar problemas de salud prioritarios. Aunque su diseño e implementación han sido destacados por diversas agencias nacionales e internacionales (incluyendo OECD), falencias a la hora de evaluar su impacto han disminuido la confianza en sus potenciales beneficios y sustentabilidad ${ }^{2}$. En particular, ha recibido limitada atención la evaluación y seguimiento de las nuevas prácticas diagnósticas y terapéuticas que introduce el GES. Indicadores básicos como el número de profesionales apropiadamente entrenados en el uso de guías clínicas, el grado de aceptación y adecuación de estas guías a los diferentes contextos sanitarios, y el nivel en que las prácticas clínicas son implementadas como sugieren las guías continúan siendo, en general, desconocidos. Robusta evidencia señala que la efectividad de intervenciones en salud depende mayoritariamente de su implementación ${ }^{3}$. Luego, la calidad de los servicios ofrecidos en el marco del GES, y en otros programas de salud, pueden estar afectados por la forma en que estos se implementan.

El desconocimiento sobre los procesos de implementación es un problema a nivel global, y uno de los puntos más críticos en el desarrollo de servicios de salud actualmente ${ }^{4}$. Aunque la evidencia respecto de técnicas diagnósticas y tratamientos para diversas patologías se acumula a un ritmo acelerado, poco se sabe respecto de las barreras y facilitadores para adoptar y sostener estas tecnologías. Los procesos de implementación suelen describirse en formato de anécdotas o estudio de casos, sin un método sistemático que considere instrumentos, estrategias, e indicadores que reflejen implementación en diferentes niveles (p.ej. individual, organizacional) y con base en diversos actores (p.ej. profesionales, pacientes) ${ }^{4}$.

En respuesta a esta problemática, ha surgido un nuevo paradigma para conceptualizar, diseñar, y evaluar servicios de salud denominado "ciencia de la implementación" o "investigación en implementación". La ciencia de la implementación busca describir, analizar e intervenir aquellos procesos y factores que se asocian a una integración efectiva de intervenciones basadas en evidencia, en un contexto clínico o comunitario en particular ${ }^{5}$. Algunas preguntas por abordar empleando este enfoque son: ¿Es posible aplicar intervenciones con base en evidencia en otros contextos? ¿Cuáles son los mejores métodos para desarrollar implementación de programas? ¿Cuáles indicadores (outcomes) e instrumentos deberían ser utilizados para determinar el nivel de implementación de un programa o intervención? ¿Cuál es la relación entre indicadores de implementación e indicadores de efectividad clínica (p.ej. reducción de síntomas, hospitalizaciones)? Estas preguntas son relevantes para el contexto nacional. $\mathrm{Su}$ respuesta permitiría contribuir al desarrollo de evaluaciones pertinentes de los actuales servicios, optimizar o reemplazar intervenciones, y orientar futuras iniciativas con mayor costo-efectividad.

La investigación en implementación sugiere modelos, estrategias, e indicadores específicos para evaluar procesos de implementación. Dichos componentes son diferentes de los indicadores clínicos, no obstante, están relacionados. Por ejemplo, modelos como el RE-AIM (Reach, Effectiveness, Adoption, Implementation, and Maintenance) y el Dynamic Adaption Process han sido propuestos para dar cuenta de las diferentes etapas de un proceso de implementación, desde la evaluación de barreras y facilitadores, hasta la adaptación de intervenciones a contextos específicos y el establecimiento de monitoreos efectivos y permanentes ${ }^{3}$. Se han sugerido, además, matrices que organizan y clasifican los diferentes tipos de estrategias para introducir una nueva práctica, considerando actores (profesional, familia, paciente), contexto (hospital, atención primaria, centro comunitario), actividad (entrenamiento presencial, 
charlas, plataformas digitales), dosis (presencial vs semi-presencial), entre otras. Además, se han desarrollado indicadores de resultados que miden el nivel de implementación, tales como aceptabilidad, adopción, fidelidad, costo, y sustentabilidad, los cuales reflejan procesos que median entre la inclusión de una nueva intervención y el resultado esperado ${ }^{5}$. Por ejemplo, el nivel de aceptación de una nueva práctica clínica por parte de profesionales puede influir si estos ofrecen o no un nuevo tratamiento, afectando, eventualmente, los potenciales beneficios en la población usuaria.

El conjunto de herramientas conceptuales y metodológicas que ofrece la Ciencia de la Implementación ofrece oportunidades para mejorar las actuales y futuras iniciativas en salud, contribuyendo a evaluar su impacto y la adopción- y por tanto la capitalización- de las transformaciones propuestas. Recientes estudios locales, como la evaluación del programa Más Adultos Mayores Autovalentes ${ }^{6}$, analizan dimensiones de validez interna de intervenciones y programas con el foco de potenciar sus resultados, utilizando una perspectiva de implementación. En consecuencia, instamos a la institucionalidad pública a considerar este nuevo paradigma que podría contribuir al inicio de una nueva ola de reformas sanitarias en el país.

Franco Mascayano ${ }^{1,2, a}$, Jean Gajardo ${ }^{3,4, c}$

${ }^{1}$ Department of Epidemiology, Mailman School of Public Health, Columbia University, New York. ${ }^{2}$ Division of Behavioral Health Services and Policy Research, New York State Psychiatric Institute, New York. ${ }^{3}$ Facultad de Medicina, Universidad San Sebastián. ${ }^{4}$ Departamento de Terapia Ocupacional y Ciencia de la Ocupación, Universidad de Chile. ${ }^{a}$ Magister en Salud Pública. 'Doctor en Salud Pública.

\section{Referencias}

1. http://www.minsal.cl/programa-de-salud-del-presidente-sebastian-pinera-una-cirugia-mayor-a-la-salud/.

2. Vergara M. Reforma del Sector de la Salud en Chile: Avances y Problemas en su Implementación. Serie Políticas Públicas. 2007 (112).

3. Brownson RC, Colditz GA, Proctor EK, editors. Dissemination and implementation research in health: translating science to practice. Oxford University Press; 2017.

4. Proctor EK, Landsverk J, Aarons G, Chambers D, Glisson C, Mittman B. Implementation research in mental health services: an emerging science with conceptual, methodological, and training challenges. Administration and Policy in Mental Health and Mental Health Services Research 2009; 36 (1): 24-34

5. Proctor E, Silmere H, Raghavan R, Hovmand P, Aarons G, Bunger A, et al. Outcomes for implementation research: conceptual distinctions, measurement challenges, and research agenda. Administration and Policy in Mental Health and Mental Health Services Research 2011; 38 (2): 65-76.

6. Gajardo J. Ampliando oportunidades para promover funcionalidad y prevenir la dependencia en población adulta mayor. Revista Med Chile 2017; 145 (6): 817-8.

Correspondencia a:

Franco Mascayano

722 West 168th St., Room 1030

New York NY 10032

fm2582@cumc.columbia.edu 